\title{
Transient global amnesia after epiduroscopy in a failed back surgery syndrome patient: Case report ${ }^{*}$
}

\author{
Leonardo Consoletti, Antonella Cotoia\#, Gilda Cinnella, Michele Dambrosio
}

Department of Anesthesiology, Critical Care and Pain Unit, University of Foggia, Foggia, Italy;

\#Corresponding Author: acotoia@libero.it

Received 14 July 2013; revised 15 August 2013; accepted 12 September 2013

Copyright (C) 2013 Leonardo Consoletti et al. This is an open access article distributed under the Creative Commons Attribution License, which permits unrestricted use, distribution, and reproduction in any medium, provided the original work is properly cited.

\begin{abstract}
Epiduroscopy (EDS) allows diagnosis and treatment of spinal nerve root pathology by a fiberoptic device inserted percutaneously in the epidural space. Transient global amnesia syndrome (TGA) is defined by an abrupt onset of an anterograde and retrograde amnesia that lasts up to $24 \mathrm{~h}$, although mild subclinical neuropsychological deficits, with concomitant vegetative symptoms, can last for days after the episode. To our knowledge no cases of TGA in association with EDS have been recognized. We present a case of a 54-year-old man who was admitted for EDS, having experienced two times the failed surgery for spinal and left leg pain. During the EDS, $10 \mathrm{ml}$ of non-ionic contrast media (CM) was injected to confirm the inside lesions. The epidural adhesions were gently broken down and drugs were injected around any inflamed nerve roots. Immediately after EDS, the patient experienced a TGA with loss of memory for the recent procedure and an impaired ability to retain new information. There were no signs of confusion or altered conscious level or seizure activities and neurological examination was normal. At $24 \mathrm{~h}$ a cranial computerized tomography (CCT) without CM showed "abnormal contrast enhancement at subarachnoid liquoral spaces levels", then at $72 \mathrm{~h} \mathrm{CCT}$ control was normal. The patient was discharged on the seventh day with perioperative lacunar amnesia persisting at this time. Herein, we sought a possible relationship between neurotoxic effect of CM and TGA in a patient emotionally stressed, even if the $C M$ dose was given as a normal clinical range.
\end{abstract}

Keywords: Intrathecal Injection of Radiopaque

\footnotetext{
*The author reports no conflicts of interest in this work.
}

Contrast; Epiduroscopy; Failed Back Surgery

Syndrome; Transient Global Amnesia; Nonionic Contrast Media

\section{INTRODUCTION}

Epiduroscopy (EDS) consists of the direct visualization of the epidural space by a percutaneously inserted fiberoptic device through the sacral hiatus which allows the simultaneously diagnosis and treatment of epidural adhesions and delivering drugs [1-5]. Direct visualization of the epidural space and contrast-enhanced fluoroscopic guidance provide complementary information and allow promptly identifying epidural fibrosis, epidural adhesions, inflammatory reaction, blood stagnation that modify the anatomy of the epidural space and disrupt tissue planes. Conditions in which EDS is indicated include failed back surgery syndrome (FBSS), a syndrome of intractable back and legs chronic pain which refers to a condition in which the symptoms remain unchanged or worsen after surgery. FBSS causes have not been thoroughly described. It has been variously attributed to adhesions in the epidural space after back surgery, physical obstructtion of the peripheral nerves, and nerve root damage [6]. Many authors reported that adhesiolysis of the epidural space and the nerve root is effective for FBSS patients [7-9]. The caudal approach to the epidural space can be safely reached via the sacral hiatus with the EDS especially under fluoroscopic control $[5,10,11]$. However, complications as direct nerve root injury, dural puncture, inadvertent entry into the subarachnoid and subdural space, neck or head pain can even occur in experienced hands [5,8,9,12].

There are no reports in the literature of transient global amnesia (TGA) after epiduroscopy performed in a patient suffering of FBSS.

\section{CASE REPORT}

A 54-year-old man was admitted for EDS, having ex- 
perienced two times the failed surgery for spinal and left leg pain (FBSS). He enjoyed good health (ASA I) except for a history of hypothyroidism, controlled with oral levothyroxine sodic. There was no past or family history of transient ischemic attack, epilepsy, migraine, or stroke. Physical examination was unremarkable and the blood chemistries were all normal. The technique used was standardized: the patient was placed in the prone position on a radiology table and, after premedication (midazolam $0.01 \mathrm{mg} / \mathrm{kg}$ and fentanyl $100 \mathrm{mcg}$ ), a local anesthetic injection was performed in and around the sacral hiatus to numb the area. A 18 Gauge needle was inserted through the sacral hiatus into the epidural space. Through this needle was then passed a fine metal guide wire. The needle was removed leaving the guide wire in place in the epidural space. A series of dilators were then passed over the guide wire until the sacral membrane accepted the sheath cannula. Once the sheath was in place, the guide wire was removed. Under fluoroscopic guidance, the epidural space was aimed via the caudal route through the Myelotec introducer's system (Myelotec, Inc., Roswell-USA). Before introducing the videoguided catheter, a $10 \mathrm{ml}$ of non ionic contrast media (CM) (IopamidolBracco, Milan, Italy) was injected to confirm the inside lesions (Figure 1(a)). Therefore a flexible fiberoptic endoscope (Myelotec, Inc., Roswell-USA) was advanced upwards using X-ray guidance, until it reached the area where epidural adhesions have been found on an magnetic resonance imaging scan. Adhesiolysis was carried out endoscopically at L5-S1 level. The endoscope was carefully advanced to the L1-L2 (area of suspected pain) without any difficulty. The endoscope was removed and a fluoroscopic control was done using CM (10 ml Iopamidol) (Figure 1(b)). Infusion was made by individual boluses of $20 \mathrm{ml}$ in order to distend the epidural space and have a good visual field during the procedure. The total volume of physiological solution infused was 160 $\mathrm{ml}$, according to the range between 50 and $250 \mathrm{ml}$ described in literature [13]. Cardiovascular and respiratory parameters ranged within normal limits during the procedure which took approximately $20 \mathrm{~min}$. Patient remained alert and communicative during the entire procedure. Immediately after, the patient experienced a global amnesia. There were no signs of confusion or altered conscious level or seizure activities. Neurological examination was normal with no evidence of any clinical focal deficit. At $24 \mathrm{~h}$ a cranial computerized tomography (cCT) without contrast injection showed "abnormal contrast enhancement at subarachnoid liquoral spaces levels” (Figure 2). Thereafter, the second cCT control at 72 $\mathrm{h}$ revealed normal finding (Figure 3) while the patient's neurological examination continued to be normal with a reduction of preoperatory pain scores and a fast recovery of identity and other memories except the perioperatory time and the mean of the procedure. The patient was discharged on the seventh day without any neurological deficit except the persistent partial amnesia referred to the $24 \mathrm{~h}$ pre/post-epiduroscopy which persists at this time.

\section{DISCUSSION}

TGA syndrome is an anterograde and retrograde amnesia with abrupt onset lasting up to $24 \mathrm{~h}$ [14]. Since 1964 the clinical characteristics of TGA have been well described. It generally occurs in persons over the age of 50 who stay alert and communicative [15]. They are disoriented in time and often in place with no loss of personal identity. The memory impairment in TGA shows a profound reduction of anterograde and a milder reduction of retrograde episodic memory, including executive functions and recognition [14,16,17]. Although the core amnestic acute phase syndrome resolves spontaneously in less than $24 \mathrm{~h}$, a subclinical impairment of memory functions might persist for months after the acute episode

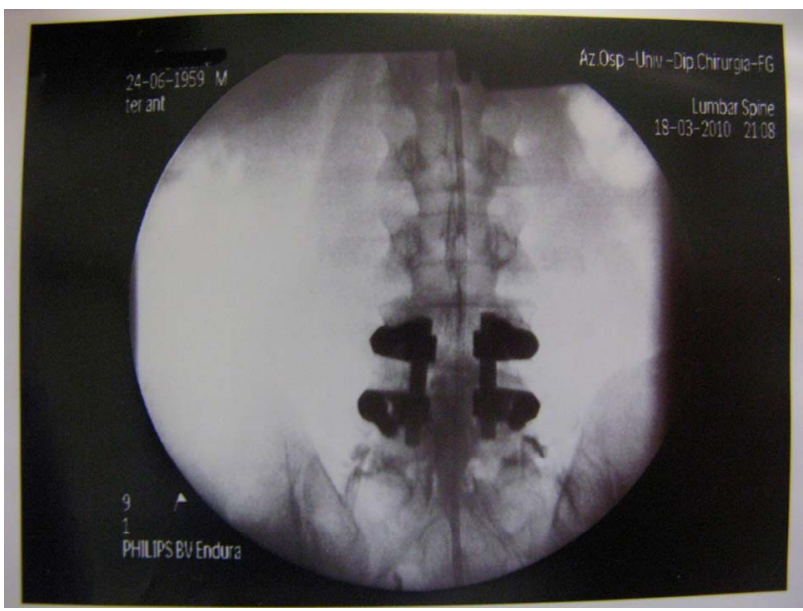

(a)

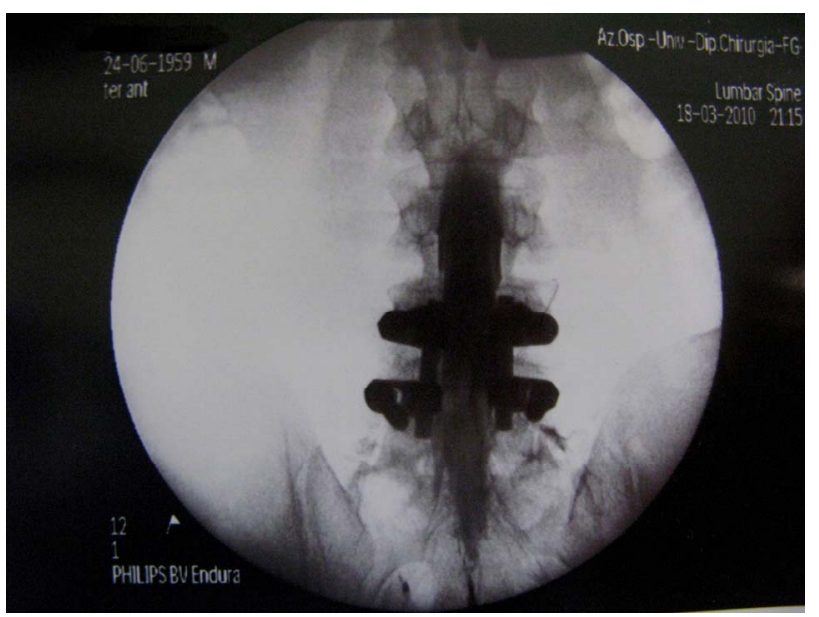

(b)

Figure 1. (a) Epidurogram showing epidural adhesions; (b) Epidurogram after epidural adhesiolysis. 


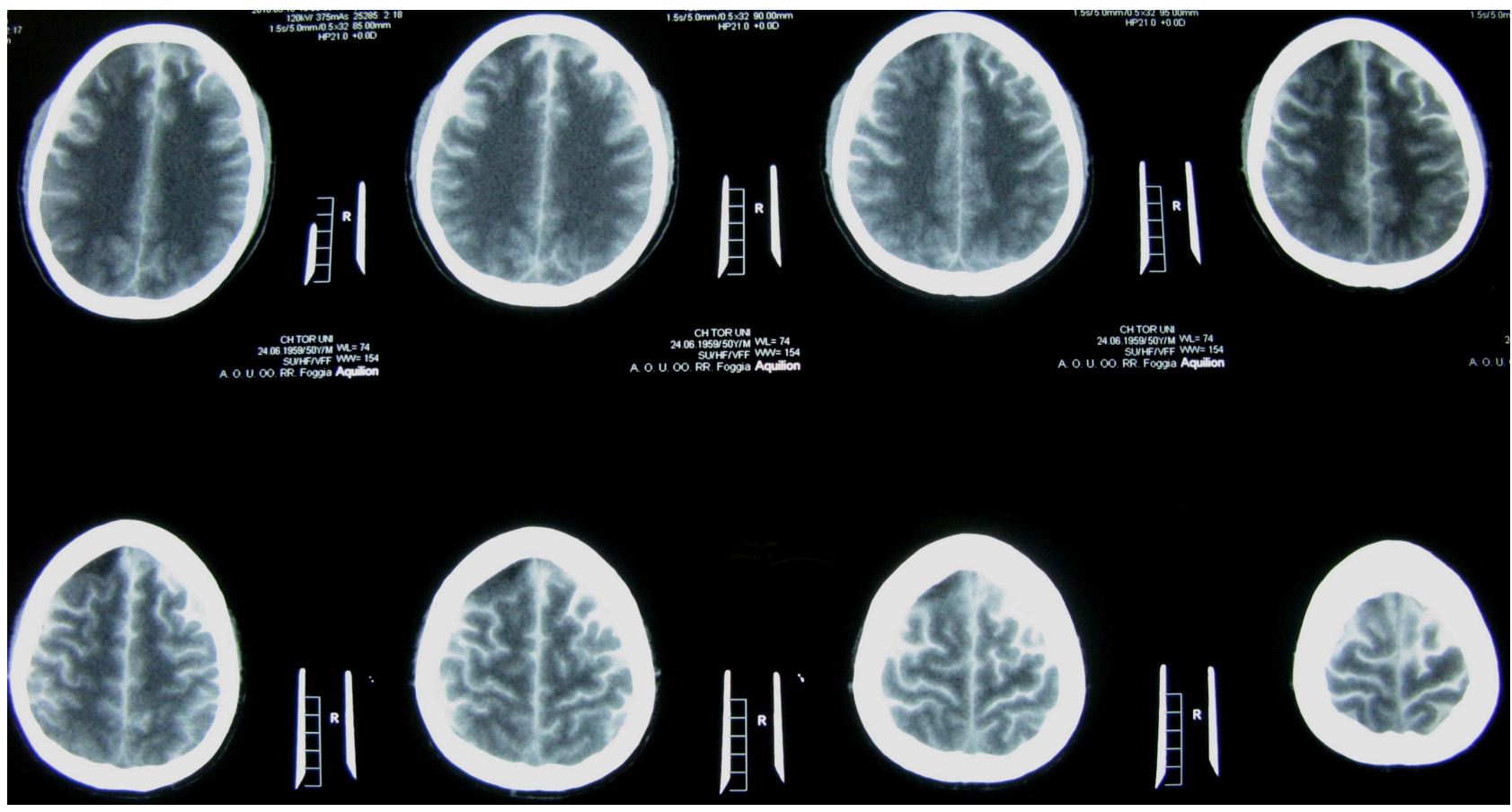

Figure 2. Cranial computerized tomography control without contrast injection at 24 h showing "abnormal contrast enhancement at subarachnoid liquoral spaces levels”.

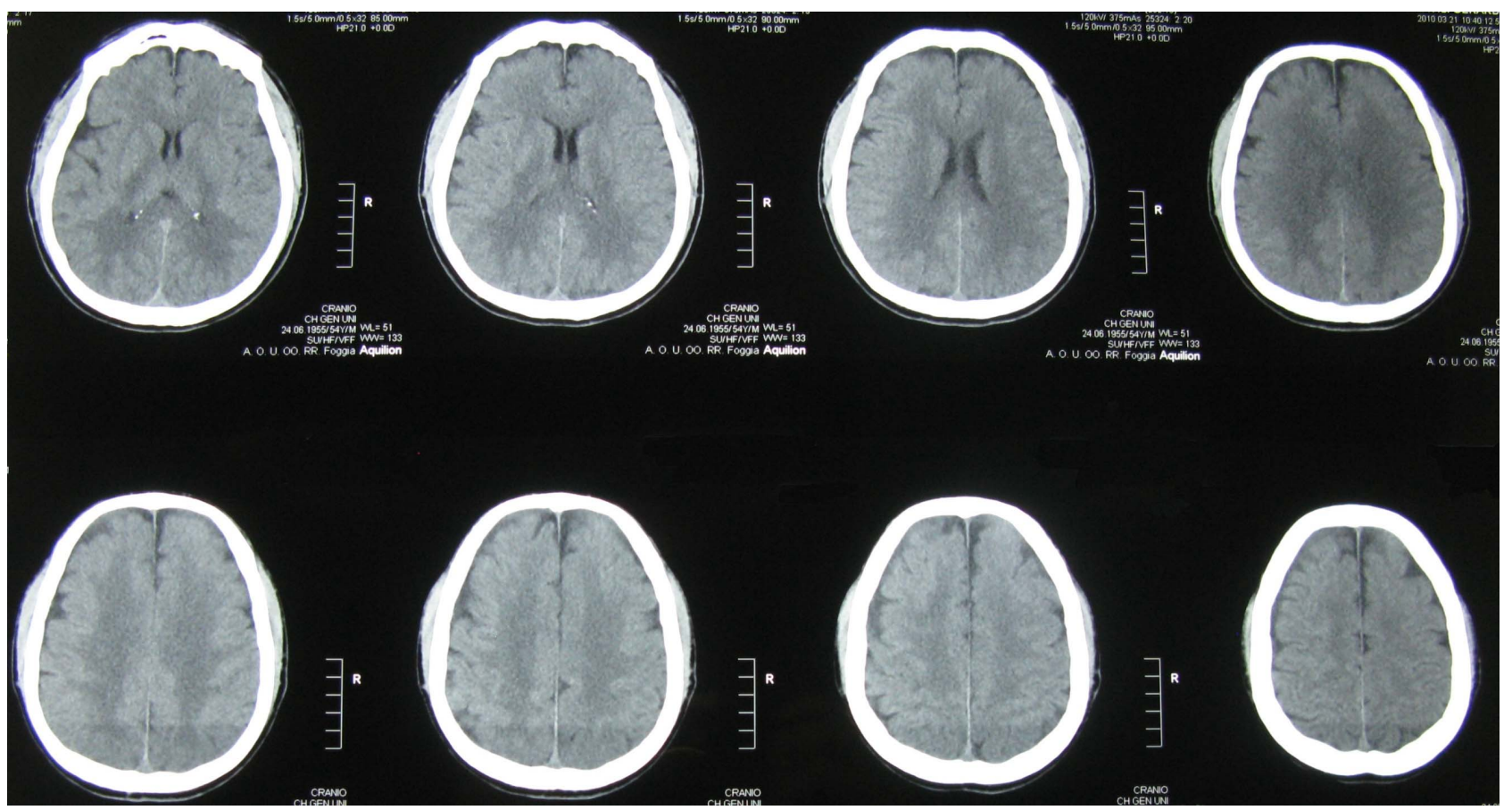

Figure 3. Cranial computerized tomography control at $72 \mathrm{~h}$ revealing normal finding.

and mild subclinical neuropsychological deficits with concomitant vegetative symptoms can last for days after the episode [14]. Currently there is no agreement on the aetiology of TGA, although several theories have been suggested. The pathogenesis of TGA might be associated of a transient perturbation of hippocampal function and different precipitating events including a stress response, emotional state, painful experiences, temperature extremes (e.g., those produced by swimming in cold water or taking a hot bath), sexual intercourse are present in $33 \%$ to $84 \%$ of attacks $[15,17-24]$. TGA has also been reported to be associated with Valsalva manoeuvre [25], 
bilateral vertebral artery dissection [26] and aortic dissection [27]. A case of TGA has been reported following the use of sildenafil [28] or ergotamine and dihydroergotamine [29]. Moreover the literature reports several cases of TGA in patients receiving CM during coronaro angiography and cerebral angiography [20,30-34]. The vast majority of recorded cases occurred as a complication of angiography with $\mathrm{CM}$. The existence of a pathogenic link between TGA and CM is unclear, even though experimental studies have suggested that CM can have neurotoxic effect altering the permeability of the bloodbrain barrier.

Herein, we sought a possible relationship between neurotoxic effect of CM and TGA in a patient emotionally stressed, even if the CM dose was given as normal clinical range (5 - $15 \mathrm{ml})$. Probably, an unsuspected dural breach occurred and the CM caught up the subarachnoid spaces (Figure 2). Differential diagnosis of TGA is with midazolam-related amnesia which is associated with anxiolytic and sedative effects, while TGA is a distinct neurological disorder characterized of sudden onset of profound amnesia anterograde and retrograde and the complete mental recovery except for the memory of episode. It presents sign of agitation, repetitive questioning and comments [35]. Therefore, we hypotize that the midazolam $0.01 \mathrm{mg} / \mathrm{kg}$ given preoperatively is not a cause of amnesia in the patient who remained alert and communicative during the entire procedure. Furthermore, midazolam-related amnesia is associated with a doseresponse curve [36] and it has been reported in the scientific literature for the use of higher doses than the one we used [37-41].

\section{CONCLUSION}

In conclusion, the existence of a pathogenic link between CM and TGA remains unclear, but our case further supports a causal relationship between them. The clinical symptoms, timeline and physical examination are helpful for the diagnosis of TGA syndrome, a dramatic event which occurs without warning. It should be promptly recognized by clinicians who have to inform patients and their family about its good prognosis.

\section{REFERENCES}

[1] Manchikanti, L., Pampati, V., Bakhit, C.E. and Pakanati, R.R. (1999) Non-endoscopic and endoscopic adhesiolysis in post-lumbar laminectomy syndrome: A one-year outcome study and cost effectiveness analysis. Pain Physician, 2, 52-58.

[2] Saberski, L.R. and Brull, S.J. (1995) Epidural endoscopyaided drug delivery: A case report. Yale Journal of Biology and Medicine, 68, 17-18.

[3] Saberski, L.R. and Kitahata, L.M. (1996) Review of the clinical basis and protocol for epidural endoscopy. Connecticut Medicine, 60, 71-73.

[4] Takeshima, N., Miyakawa, H., Okuda, K., Hattori, S., Hagiwara, S., Takatani, J., et al. (2009) Evaluation of the therapeutic results of epiduroscopic adhesiolysis for failed back surgery syndrome. British Journal of Anaesthesia, 102, 400-407. http://dx.doi.org/10.1093/bja/aen383

[5] Geurts, J.W., Kallewaard, J.W., Richardson, J. and Groen, G.J. (2002) Targeted methylprednisolone acetate/hyaluronidase/clonidine injection after diagnostic epiduroscopy for chronic sciatica: A prospective, 1-year follow-up study. Regional Anesthesia and Pain Medicine, 27, 343-352.

[6] Frocrain, L., Duvauferrier, R., Husson, J.L., Noel, J., Ramee, A. and Pawlotsky, Y. (1989) Recurrent postoperative sciatica: Evaluation with MR imaging and enhanced CT. Radiology, 170, 531-533.

[7] Chopra, P., Smith, H.S., Deer, T.R. and Bowman, R.C. (2005) Role of adhesiolysis in the management of chronic spinal pain: A systematic review of effectiveness and complications. Pain Physician, 8, 87-100.

[8] Trescot, A.M., Chopra, P., Abdi, S., Datta, S. and Schultz, D.M. (2007) Systematic review of effectiveness and complications of adhesiolysis in the management of chronic spinal pain: an update. Pain Physician, 10, 129-146.

[9] Manchikanti, L., Boswell, M.V., Datta, S., Fellows, B., Abdi, S., Singh, V., et al. (2009) Comprehensive review of therapeutic interventions in managing chronic spinal pain. Pain Physician, 12, E123-E198.

[10] Saberski, L.R. and Kitahata, L.M. (1995) Direct visualization of the lumbosacral epidural space through the sacral hiatus. Anesthesia \& Analgesia, 80, 839-840.

[11] Saberski, L.R. and Gerena, F. (1998) Safety of epidural endoscopy. Regional Anesthesia and Pain Medicine, 23, 324-325. http://dx.doi.org/10.1016/S1098-7339(98)90065-7

[12] Manchikanti, L. and Singh, V. (2002) Epidural lysis of adhesions and myeloscopy. Current Pain and Headache Reports, 6, 427-435. http://dx.doi.org/10.1007/s11916-002-0061-2

[13] Van Boxem, K., Cheng, J., Patijn, J., van Kleef, M., Lataster, A., Mekhail, N., et al. (2010) 11. Lumbosacral radicular pain. Pain Practice, 10, 339-358. http://dx.doi.org/10.1111/j.1533-2500.2010.00370.x

[14] Bartsch, T. and Deuschl, G. (2010) Transient global amnesia: Functional anatomy and clinical implications. Lancet Neurology, 9, 205-214. http://dx.doi.org/10.1016/S1474-4422(09)70344-8

[15] Hodges, J.R. and Warlow, C.P. (1990) Syndromes of transient amnesia: Towards a classification. A study of 153 cases. Journal of Neurology, Neurosurgery \& Psychiatry, 53, 834-843. http://dx.doi.org/10.1136/jnnp.53.10.834

[16] Fisher, C.M. and Adams, R.D. (1964) Transient global amnesia. Acta Neurologica Scandinavica, 40, 1-83.

[17] Berli, R., Hutter, A., Waespe, W. and Bachli, E.B. (2009) Transient global amnesia-Not so rare after all. Swiss Medical Weekly, 139, 288-292.

[18] Kessler, J., Markowitsch, H.J., Rudolf, J. and Heiss, W.D. (2001) Continuing cognitive impairment after isolated 
transient global amnesia. International Journal of Neuroscience, 106, 159-168. http://dx.doi.org/10.3109/00207450109149746

[19] Merriam, A.E., Wyszynski, B. and Betzler, T. (1992) Emotional arousal-induced transient global amnesia. A clue to the neural transcription of emotion? Psychosomatics, 33, 109-113. http://dx.doi.org/10.1016/S0033-3182(92)72029-5

[20] Minuk, J., Melancon, D., Tampieri, D. and Ethier, R. (1990) Transient global amnesia associated with cerebral angiography performed with use of iopamidol. Radiology, 174, 285-286.

[21] Bucuk, M., Muzur, A., Willheim, K., Jurjevic, A., Tomic, Z. and Tuskan-Mohar, L. (2004) Make love to forget: Two cases of transient global amnesia triggered by sexual intercourse. Collegium Antropologicum, 28, 899-905.

[22] Frederiks, J.A. (1993) Transient global amnesia. Clinical Neurology and Neurosurgery, 95, 265-283. http://dx.doi.org/10.1016/0303-8467(93)90102-M

[23] Miller, J.W., Petersen, R.C., Metter, E.J., Millikan, C.H. and Yanagihara, T. (1987) Transient global amnesia: Clinical characteristics and prognosis. Neurology, 37, 733737. http://dx.doi.org/10.1212/WNL.37.5.733

[24] Agosti, C., Akkawi, N.M., Borroni, B. and Padovani, A. (2006) Recurrency in transient global amnesia: A retrospective study. European Journal of Neurology, 13, 986989. http://dx.doi.org/10.1111/j.1468-1331.2006.01408.x

[25] Sander, D., Winbeck, K., Etgen, T., Knapp, R., Klingelhofer, J. and Conrad, B. (2000) Disturbance of venous flow patterns in patients with transient global amnesia. Lancet, 356, 1982-1984. http://dx.doi.org/10.1016/S0140-6736(00)03313-4

[26] Michel, D., Garnier, P., Schneider, F., Poujois, A., Barral, F.G. and Thomas-Anterion, C. (2004) Diffusion MRI in pure transient global amnesia associated with bilateral vertebral artery dissection. Cerebrovascular Diseases, 17, 264-266. http://dx.doi.org/10.1159/000076964

[27] Mondon, K., Blechet, C., Gochard, A., Elaroussi, D., Fetissof, F., De Toffol, B., et al. (2007) Transient global amnesia caused by painless aortic dissection. Emergency Medicine Journal, 24, 63-64. http://dx.doi.org/10.1136/emj.2006.040881

[28] Savitz, S.A. and Caplan, L.R. (2002) Transient global amnesia after sildenafil (Viagra) use. Neurology, 59, 778. http://dx.doi.org/10.1212/WNL.59.5.778

[29] Gil-Martinez, T. and Galiano, R. (2004) [Transient global amnesia following the use of ergots in the treatment of migraine]. Revista de Neurología, 39, 929-931.

[30] Kurokawa, Y., Ishizaki, E., Kihara, H. and Inaba, K. (2004) [Two cases of transient global amnesia (TGA) immedi- ately after coronary angiography: Pathogenesis of the primary TGA]. No To Shinkei, 56, 69-74.

[31] Meder, J.F., Mourey-Gerosa, I., Blustajn, J., Lemaignen, H., Devaux, B. and Fredy, D. (1997) Transient global amnesia after cerebral angiography. A case report. Acta Radiologica, 38, 273-274.

[32] Jackson, A., Stewart, G., Wood, A. and Gillespie, J.E. (1995) Transient global amnesia and cortical blindness after vertebral angiography: Further evidence for the role of arterial spasm. American Journal of Neuroradiology, 16, 955-959.

[33] Juni, J., Morera, J., Lainez, J.M., Escudero, J., Ferrer, C. and Sancho, J. (1992) Transient global amnesia after cerebral angiography with iohexol. Neuroradiology, 34, 141143. http://dx.doi.org/10.1007/BF00588161

[34] Giang, D.W. and Kido, D.K. (1989) Transient global amnesia associated with cerebral angiography performed with use of iopamidol. Radiology, 172, 195-196.

[35] Ghoneim, M.M. (1998) Transient global amnesia: A cause for postanesthetic memory disorder. Anesthesia \& Analgesia, 87, 980-981.

[36] Vinik, H.K.I. Midazolam induced amnesia: A dose response curve and enhancement by alfentanil in a double-blind, randomized study. Memory and Awareness in Anesthesia.

[37] Koyama, S., Ohashi, N., Kurita, S., Nakatani, K., Nagata, N. and Toyoda, Y. (2008) [Conscious sedation and amnesic effect of intravenous low-dose midazolam prior to spinal anesthesia]. Masui, 57, 713-718.

[38] Stevanovic, P. (2006) [Midazolam (dormicump)-Clinical practice guidelines]. Medicinski Pregled, 59, 89-94. http://dx.doi.org/10.2298/MPNS0602089S

[39] Kain, Z.N., Hofstadter, M.B., Mayes, L.C., Krivutza, D. M., Alexander, G., Wang, S.M., et al. (2000) Midazolam: Effects on amnesia and anxiety in children. Anesthesiology, 93, 676-684. http://dx.doi.org/10.1097/00000542-200009000-00016

[40] Veselis, R.A., Reinsel, R., Alagesan, R., Heino, R. and Bedford, R.F. (1991) The EEG as a monitor of midazolam amnesia: Changes in power and topography as a function of amnesic state. Anesthesiology, 74, 866-874. http://dx.doi.org/10.1097/00000542-199105000-00011

[41] Nakasuji, M., Nakamura, M., Imanaka, N., Tanaka, M., Nomura, M., Wada, M., et al. (2009) Bispectral index during epidural puncture predicts anterograde amnesia in patients given midazolam premedication. Journal of Anesthesia, 23, 329-333. http://dx.doi.org/10.1007/s00540-009-0748-1

Non-ionic contrast media (CM)

Cranial computerized tomography (cCT)

Failed back surgery syndrome (FBSS)

\section{Epiduroscopy (EDS)}

LIST OF ABBREVIATIONS

Transient global amnesia syndrome (TGA) 